\title{
Early post-STEMI PET, a judicious investment?
}

\author{
Riemer H. J. A. Slart, MD, PhD, ${ }^{\mathrm{a}, \mathrm{b}}$ and Luis Eduardo Juarez-Orozco, $\mathrm{MD}, \mathrm{PhD}^{\mathrm{c}}$ \\ a Medical Imaging Center, Department of Nuclear Medicine \& Molecular Imaging, University of \\ Groningen, University Medical Center Groningen, Groningen, The Netherlands \\ b Department of Biomedical Photonic Imaging, University of Twente, Enschede, The Netherlands \\ c Turku PET Centre, Turku University Hospital, Turku, Finland
}

Received Jul 24, 2017; accepted Jul 24, 2017

doi: $10.1007 / \mathrm{s} 12350-017-1015-8$

\section{See related article, pp. 471 -482}

Improvements in the management of acute myocardial infarction together with population aging have made heart failure a growing burden to health care. This improved management originates from an optimized clinical approach to acute coronary syndromes and the efficacy of percutaneous coronary intervention (PCI). Both factors have contributed to the reduction of acute mortality in patients faced with ST-segment elevation myocardial infarction (STEMI). ${ }^{1}$ In this setting, a challenge has arisen in identifying those patients who are at risk of progressive adverse remodeling and myocardial dysfunction. This challenge now constitutes one of the driving forces in cardiovascular clinical research.

Parallel to this development, the arsenal of noninvasive imaging techniques at our disposal has been expanded by the development of cardiac PET and cardiac MR (CMR), which are powerful non-invasive imaging techniques conveying complementary (patho)physiological and anatomical characterization. However, since many different measurements can be performed with these techniques, which one should be applied? For instance, "list-mode" acquired PET can evaluate myocardial perfusion (reserve) in relative or absolute terms, as well as ventricular function. CMR is able to document the presence of scar tissue (through

Reprint requests: Riemer H. J. A. Slart, MD, PhD, Medical Imaging Center, Department of Nuclear Medicine \& Molecular Imaging, University of Groningen, University Medical Center Groningen, Hanzeplein 1, P.O. Box 30001, 9700 RB Groningen, The Netherlands; r.h.j.a.slart@umcg.nl

J Nucl Cardiol 2019;26:483-5.

$1071-3581 / \$ 34.00$

Copyright (c) 2017 American Society of Nuclear Cardiology. late gadolinium enhancement [LGE]), myocardial perfusion (through the use of gadolinium in the first pass), and even tissue edema (through T2-weighted imaging), while providing accurate functional evaluation (through cine imaging) of the myocardium in the form of ventricular dimensions and ejection fraction, as well as wall motion and thickening.

With this in mind, a number of studies have demonstrated that deteriorating ventricular dimensions (known as adverse remodeling) and function after a hard event, such as STEMI, constitute important predictors of the risk for developing major adverse cardiovascular events, including cardiac death and heart failure. ${ }^{2}$ These studies, however, have been performed after a stabilization period of weeks or months, and our understanding of early (subacute) predictors of adverse remodeling is still incomplete.

In the current issue of the Journal of Nuclear Cardiology, Ghotbi and colleagues propose rest ${ }^{82} \mathrm{Rb}$ PET imaging for the evaluation of myocardial perfusion in the subacute (early) phase after a PCI-treated STEMI, and evaluate its predictive relationship with ulterior CMRassessed ventricular remodeling and function after 3 months. ${ }^{3}$ Although a relatively small number of patients were examined ( 35 subjects), their study is a valuable investigation. PET myocardial perfusion imaging has proven to have superior image quality to SPECT, and it represents the current reference standard to quantify myocardial blood flow, ${ }^{4}$ both globally and segmentally. PET has constitutional advantages over SPECT such as constitutional attenuation correction, higher spatial resolution, and better image contrast. The availability of fast photomultipliers, high-density scintillators, modern electronics, and faster computational capabilities, including time-of-flight systems, has made it possible to optimize perfusion imaging. ${ }^{5}$ Thus, PET provides the best option for accurately characterizing the myocardial perfusion status. Because of the clinical setting of the study by Ghotbi et al, PET was performed only during rest for 
reasons of safety in order to refrain patients from undergoing a pharmacological stress test. Thus, the traditional two-phase PET perfusion scan (which allows for myocardial perfusion reserve evaluation) and the regionally proposed stress-only approach $^{6}$ were not applied. Nevertheless, this rest-only scanning can be of interest as a particular application of cardiac PET, since it has the additional advantage of a reduced radiation dose and therefore, warrants further research. However, additional insight into the influence of well-known factors that influence myocardial blood flow at rest (e.g., hypertension and gender $)^{7}$ is mandatory. An interesting feature in gated cardiac PET is that it allows the reconstruction of ECGgated datasets for simultaneous functional analysis that provides adequate wall motion scores as well as left ventricular volume and ejection fraction (LVEF). In fact, it has been documented that the prognostic value of ${ }^{82} \mathrm{Rb}$ PET for all-cause mortality can be enhanced, by adding PET-measured LVEF data. ${ }^{8}$ Hence, the parallel evaluation of both myocardial perfusion and function with PET early after STEMI should also be considered ${ }^{9}$ for optimal assessment of myocardial viability.

The main contribution of this Danish study is the documented predictive link between segmental PET perfusion findings, at a median of 36 hours after PCI, and CMR functional and morphological parameters at follow-up. ${ }^{3}$ In particular, the extent of the relative perfusion defect (as a surrogate for area of infarction) was significantly associated with ventricular function measured by wall thickening $(\beta=-0.36, P=.001)$ and LGE $(\beta=0.43, P=.001)$, and, to a smaller degree, with wall motion $(\beta=-0.04, \quad P=.001)$ after 3 months. Similar results were found for absolute myocardial blood flow (wall thickening: $\beta=39.6$, $P=.001$; LGE: $\beta=4.6, P=.001$; wall motion: $\beta=-38.4, P=.001$ ). In terms of remodeling (ventricular morphology) at follow-up, the extent of the perfusion defect was correlated with LVEF (at the expense of end-systolic volume) and final infarct size ( $r=-0.53$ and $r=0.58$, respectively). However, these correlations were considerably moderate compared with absolute myocardial blood flow $(r=0.41$ and $r=$ -0.32 ). Whether the timing of PET imaging, the variation in ventricular segmentation, differences in the medication received (as only one-third of the patients used ACE inhibitors or ARAs at follow-up), or residual microvascular dysfunction and stunning (as pointed out by the authors) may play a role in weakening the aforementioned correlations, is a matter that should encourage future research and discussion.

Ghotbi et al utilized ${ }^{82} \mathrm{Rb}$ as a perfusion agent. ${ }^{3}$ Although myocardial perfusion can be quantified with this radiotracer, it has been demonstrated that the kinetics of ${ }^{82} \mathrm{Rb}$ lead to underestimation of myocardial blood flow at higher flow values due to a marked roll-off effect. ${ }^{10}$ Therefore, it is likely that the application of alternative tracers with more favorable extraction profiles, such as ${ }^{13} \mathrm{~N}$-ammonia and ${ }^{15} \mathrm{O}$-water, could enhance the prognostic value of PET measurements of myocardial perfusion at rest in forecasting CMR-evaluated wall motion abnormalities and scar extension. ${ }^{11-14}$ It is true, as the authors correctly state, that ${ }^{82} \mathrm{Rb}$ offers the important advantage of independence from an onsite cyclotron. However, ${ }^{13} \mathrm{~N}$-ammonia- and ${ }^{15} \mathrm{O}$-waterproducing "baby" cyclotrons are now commercially available and offer new opportunities. Dedicated software programs should also be taken into consideration for calculation of reliable all-purpose cut-off perfusion values, because of the intrinsic variability between tracers and different camera systems. ${ }^{15}$

Finally, the costs and investments related to PET imaging, as well as existing reimbursement policies, have represented constant challenges to the development and refinement of the clinical implementation of cardiac PET. In the future, when the practical utility and costeffectiveness of PET imaging have been demonstrated, further clinical implementation can be expected. Measurement of myocardial perfusion early post-STEMI may be one of the candidates for such implementation and further evidence for the clinical value of this technique is definitely desired.

\section{Disclosure}

The authors have nothing to disclose.

\section{References}

1. Keeley EC, Boura JA, Grines CL. Primary angioplasty versus intravenous thrombolytic therapy for acute myocardial infarction: A quantitative review of 23 randomised trials. Lancet. 2003;361:13-20.

2. El Aidi H, Adams A, Moons KGM, Den Ruijter HM, Mali WPTM, Doevendans PA, et al. Cardiac magnetic resonance imaging findings and the risk of cardiovascular events in patients with recent myocardial infarction or suspected or known coronary artery disease: A systematic review of prognostic studies. J Am Coll Cardiol. 2014;63:1031-45.

3. Ghotbi AA, Hasbak P, Nepper-Christensen L, Lønborg J, Atharovski $\mathrm{K}$, Christensen $\mathrm{T}$, et al. Early risk stratification using rubidium-82 positron emission tomography in STEMI patients. J Nucl Cardiol. 2017. doi:10.1007/s12350-017-0993-x.

4. Slomka P, Berman DS, Alexanderson E, Germano G. The role of PET quantification in cardiovascular imaging. Clin Transl Imaging. 2014;2:343-58.

5. Vandenberghe S, Mikhaylova E, D'Hoe E, Mollet P, Karp JS. Recent developments in time-of-flight PET. EJNMMI Phys. 2016;3(1):3.

6. Schindler TH, Dilsizian V. PET-determined hyperemic myocardial blood flow: Further progress to clinical application. J Am Coll Cardiol. 2014;64:1476-8. 
7. Joutsiniemi E, Saraste A, Pietila M, Maki M, Kajander S, Ukkonen $\mathrm{H}$, et al. Absolute flow or myocardial flow reserve for the detection of significant coronary artery disease? Eur Heart J Cardiovasc Imaging. 2014;15:659-65.

8. Lertsburapa K, Ahlberg AW, Bateman TM, Katten D, Volker L, Cullom SJ, et al. Independent and incremental prognostic value of left ventricular ejection fraction determined by stress gated rubidium 82 PET imaging in patients with known or suspected coronary artery disease. J Nucl Cardiol. 2008;15:745-53.

9. Juarez-Orozco LE. The integrative value of myocardial perfusionfunction imaging with $13 \mathrm{~N}$-ammonia positron emission tomography. Doctor of Philosophy 2017, Rijksuniversiteit Groningen, The Netherlands.

10. Nakazato R, Berman DS, Alexanderson E, Slomka P. Myocardial perfusion imaging with PET. Imaging Med. 2013;5:35-46.

11. Tio RA, Dabeshlim A, Siebelink H-MJ, de Sutter J, Hillege HL, Zeebregts CJ, et al. Comparison between the prognostic value of left ventricular function and myocardial perfusion reserve in patients with ischemic heart disease. J Nucl Med. 2009;50:214-9.

12. Slart RHJA, Zeebregts CJ, Hillege HL, de Sutter J, Dierckx RAJO, van Veldhuisen DJ, et al. Myocardial perfusion reserve after a PET-driven revascularization procedure: A strong prognostic factor. J Nucl Med. 2011;52:873-9.

13. Herzog BA, Husmann L, Valenta I, Gaemperli O, Siegrist PT, Tay $\mathrm{FM}$, et al. Long-term prognostic value of $13 \mathrm{~N}$-ammonia myocardial perfusion positron emission tomography added value of coronary flow reserve. J Am Coll Cardiol. 2009;54:150-6.

14. Murthy VL, Naya M, Foster CR, Hainer J, Gaber M, Di Carli G, et al. Improved cardiac risk assessment with noninvasive measures of coronary flow reserve. Circulation. 2011;124:2215-24.

15. Nesterov SV, Deshayes E, Sciagrà R, Settimo L, Declerck JM, Pan $\mathrm{X}-\mathrm{B}$, et al. Quantification of myocardial blood flow in absolute terms using (82)Rb PET imaging: The RUBY-10 study. J Am Coll Cardiol Img. 2014;7:1119-27. 